\section{Rendimiento de los cuestionarios de sueño en el diagnóstico de síndrome de apneas obstructivas del sueño en población chilena. Subestudio de la Encuesta Nacional de Salud, 2016/17}

\author{
FERNANDO SALDÍAS PEÑAFIEL ${ }^{1}$, PABLO BROCKMANN VELOSO ${ }^{2}$, \\ JULIA SANTÍN MARTÍNEZ ${ }^{3}$, EDUARDO FUENTES-LÓPEZ ${ }^{4}$, \\ GONZALO VALDIVIA CABRERA ${ }^{5}$
}

\section{Performance of sleep questionnaires for the diagnosis of obstructive sleep apnea syndrome}

Background: The diagnosis of obstructive sleep apnea syndrome (OSAS) is based on nocturnal records: polysomnography or respiratory polygraphy. However, their high costs limit their use. Aim: To examine the predictive value of three sleep questionnaires (STOP, STOP-Bang, Epworth Sleepiness Scale (ESS) in the screening of OSAS in Chilean adults. Material and Methods: During the National Health Survey 2016/17, 205 adults aged $50.7 \pm 15.0$ years (46\% males) living in the Metropolitan Region answered sleep questionnaires and underwent an ambulatory respiratory polygraphy. The sensitivity, specificity, positive and negative predictive values and receiver operating characteristic curves of sleep questionnaires were calculated. Results: Fifty nine percent of participants had OSAS which was moderate to severe in $26 \%$. The clinical variables associated with OSAS were age, male gender, hypertension, dyslipidemia, overweight, cervical and waist circumferences, history of regular snoring and witnessed apneas. Daytime somnolence, insomnia and unrefreshing sleep were not associated to OSAS risk. STOP, STOP-Bang and ESS questionnaires classified 64\%, 71\% and $12 \%$ of cases as high risk for OSAS, respectively. The STOP and STOPBang questionnaires had the highest sensitivity to predict OSAS (76\% and 89\%, respectively) while the ESS had the highest specificity (91\%). Conclusions: The sleep questionnaires allowed to identify the subjects at high risk for OSAS in this sample of adults from the Metropolitan Region.

(Rev Med Chile 2019; 147: 1543-1552)

Key words: Diagnosis; Sleep Apnea, Obstructive; Surveys and Questionnaires.

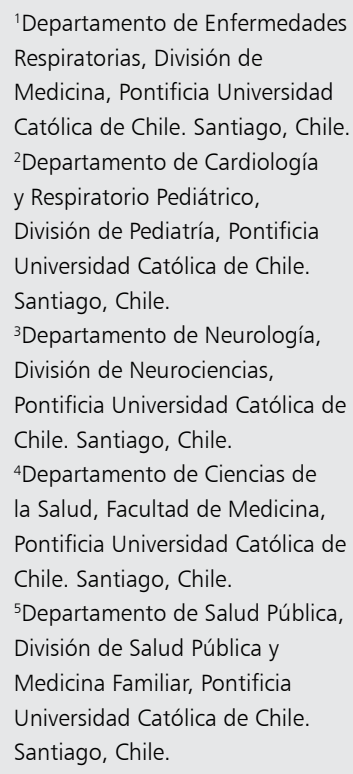

Trabajo no recibió financiamiento. Los autores declaran no tener conflictos e interés.

Recibido el 5 de julio de 2019, aceptado el 11 de diciembre de 2019.

Correspondencia a:

Dr. Fernando Saldías Peñafiel Departamento de Enfermedades Respiratorias. División de Medicina, Facultad de Medicina. Pontificia Universidad Católica de Chile

Teléfonos: (562) 26331541 (562) 23543242

Diagonal Paraguay 362. Sexto

Piso. Santiago, Chile.

fsaldias@med.puc.cl
1 1 síndrome de apneas hipopneas obstructivas del sueño (SAHOS) constituye un problema clínico prevalente que ocasiona morbilidad y mortalidad significativas en la población adul$\mathrm{ta}^{1-4}$. En los estudios poblacionales, se estima una prevalencia de SAHOS de 22\% (rango: 9-37\%) en varones y $17 \%$ (rango: $4-50 \%$ ) en mujeres.
La prevalencia de SAHOS aumenta con la edad en varones y en la mujer después de la menopausia, se asocia al índice de masa corporal y la obesidad central o visceral ${ }^{6}$. Las principales manifestaciones clínicas asociadas a esta entidad son ronquido intenso, pausas respiratorias durante el sueño, somnolencia diurna excesiva, sueño poco 
reparador, deterioro cognitivo y aumento de la accidentabilidad ${ }^{1-4}$. Los trastornos respiratorios del sueño de magnitud moderada-severa se han asociado a complicaciones cardiovasculares, tales como hipertensión arterial refractaria, fibrilación auricular paroxística, cardiopatía coronaria, insuficiencia cardiaca congestiva, síndrome metabólico $\mathrm{y}$ accidentes cerebrovasculares isquémicos ${ }^{7-12}$. El diagnóstico definitivo requiere la realización de una polisomnografía (PSG) en el laboratorio de sueño (patrón de referencia) o poligrafía respiratoria (PR) en el hogar en los pacientes sin enfermedades cardiovasculares o respiratorias crónicas con elevada sospecha de SAHOS ${ }^{1-4}$. Los costos y la complejidad técnica asociados a los exámenes han limitado el acceso de la población al diagnóstico y tratamiento oportuno de esta condición, lo cual conlleva a mayor morbilidad y mortalidad asociadas a esta entidad clínica.

Con el propósito de facilitar la pesquisa de pacientes con SAHOS en la población general, se ha examinado el rendimiento de diversas reglas clínicas predictivas basadas en elementos de la historia clínica y el examen físico ${ }^{13-21}$. El desempeño de las reglas predictivas ha sido variable, dependiendo de las características de la población examinada (edad, sexo, etnia, comorbilidades), lugar de procedencia (población general, clínicas de sueño), criterios diagnósticos y equipos empleados (PSG en el laboratorio o el hogar, PR en el laboratorio o el hogar, equipos simplificados ${ }^{22,23}$. En las guías de práctica clínica no se recomienda establecer el diagnóstico de SAHOS basados exclusivamente en elementos de la historia clínica y examen físico, debido a la falta de precisión de los síntomas y signos en la pesquisa de esta condición ${ }^{24}$.

Los cuestionarios de sueño y las reglas predictivas han sido empleados en los estudios epidemiológicos poblacionales con el propósito de identificar a los pacientes con mayor riesgo de SAHOS en la población general ${ }^{23}$. En el estudio Platino, realizado en población mayor de 40 años de la Región Metropolitana, empleando un cuestionario de sueño se estimó una prevalencia de SAHOS alrededor de 6,4\%, correspondiendo a $8,8 \%$ en los varones y $5 \%$ en las mujeres ${ }^{25}$. En la Encuesta Nacional de Salud 2009/10, empleando un cuestionario de sueño, se estimó una prevalencia de SAHOS de 4,6\% en población mayor de 15 años, correspondiendo a 5,3\% en los varones y $3,9 \%$ en las mujeres ${ }^{26}$.
El propósito del estudio es examinar el desempeño de los cuestionarios de sueño en una muestra poblacional seleccionada de la Región Metropolitana de la Encuesta Nacional de Salud 2016/17 (ENS 2016/17) a quienes se realizó poligrafía respiratoria en el hogar.

\section{Sujetos y Métodos}

Estudio transversal analítico que examinó el rendimiento de los cuestionarios de sueño en la pesquisa de SAHOS en una muestra poblacional (n: 205 sujetos) seleccionada entre los residentes de la Región Metropolitana que participaron en la Encuesta Nacional de Salud 2016-2017. La ENS 2016/17 corresponde a un estudio transversal realizado en base a la selección de una muestra aleatoria compleja, estratificada y multietápica por conglomerados, de 9.901 viviendas. La muestra fue diseñada con estratos geográficos y demográficos homogéneos lo que implicó la sobrerrepresentación de regiones distintas a la Metropolitana, zonas rurales y adultos mayores. Estas modificaciones fueron compensadas en el momento de los análisis, tomando en consideración factores de expansión que consideran las probabilidades de selección en cada etapa, corrección por no respuesta y postestratificación demográfica consecuente con las proyecciones censales de la población chilena a junio de 2016. La encuesta tuvo una tasa de respuesta en la población elegible de $67 \%$ y la tasa de rechazo fue $9,8 \% .6 .233$ sujetos mayores de 15 años respondieron a los cuestionarios que examinaron los principales problemas de salud de la población, incluyendo los trastornos respiratorios del sueño, durante la primera visita a la vivienda. 5.520 sujetos fueron examinados y entrevistados por una enfermera capacitada en la segunda visita ( $89 \%$ de la muestra) y se obtuvieron muestras de laboratorio para estudio metabólico en 5.451 participantes, midiendo la glicemia, $\mathrm{Hb}$ glicosilada, colesterol total, HDL y LDL colesterol, triglicéridos, $\mathrm{TSH}_{\text {y }} \mathrm{T}_{4}$ libre séricas, entre otras determinaciones.

En una submuestra de la Región Metropolitana, estratificada por factores de riesgo de SAHOS (ronquido habitual y pausas respiratorias presenciadas por testigos), se evaluaron 205 sujetos mayores de 18 años, a quienes se les realizó un cuestionario de sueño, mediciones antropométricas y una poligrafía respiratoria en el hogar. El 
estudio fue revisado y aprobado por el Comité de Ética en Investigación de la Facultad de Medicina de la Pontificia Universidad Católica de Chile.

En los sujetos que aceptaron participar en el estudio y firmaron el consentimiento informado se consignaron los siguientes antecedentes: edad, sexo, ocupación, nivel educacional, comorbilidades, consumo de tabaco, alcohol y fármacos, peso, talla, índice de masa corporal, perímetro cervical, circunferencia de cintura, presión arterial en posición sentada, historia de sueño (duración, presencia de ronquido, pausas respiratorias, somnolencia diurna, insomnio, sueño poco reparador) y la magnitud de la somnolencia diurna fue cuantificada con la escala de somnolencia de Epworth ${ }^{27}$. Las variables clínicas incluidas en los cuestionarios de sueño STOP $^{20}$ y STOP-Bang ${ }^{21}$ nos permiten predecir el riesgo de SAHOS en diferentes contextos clínicos ${ }^{28}$. Los sujetos con riesgo elevado de SAHOS responden afirmativamente al menos a dos (STOP) o tres (STOP-Bang) preguntas de los cuestionarios.

\section{Estudio trastornos respiratorios del sueño}

La evaluación de los trastornos respiratorios del sueño se realizó en el domicilio de los sujetos enrolados con un cuestionario de sueño y un equipo de poligrafía respiratoria que cumple con las exigencias de la Academia Americana de Medicina del Sueño (AASM) para los estudios de nivel III ${ }^{29-31}$. El equipo mide el flujo aéreo oronasal, esfuerzo respiratorio torácico y abdominal, pulsioximetría, frecuencia cardiaca, posición corporal y ronquido. Se empleó el software sleepware G3 with integrated somnolyzer scoring solution en la lectura de los exámenes. La entrega y retiro de los dispositivos fue realizado por un profesional de salud, entrenado y capacitado para esta función, quien además corroboró in situ, la existencia y adecuación del registro para su posterior lectura. El análisis de la PR fue realizado manualmente por un médico especialista en trastornos respiratorios del sueño que desconocía los antecedentes clínicos y los resultados de los cuestionarios de sueño; consignando la duración del estudio, posición corporal, número de apneas e hipopneas obstructivas, centrales y mixtas, caída de la saturación arterial de oxígeno bajo 90\% (CT90\%) y número de episodios de desaturación mayor de $4 \%$. Se calculó el número de eventos respiratorios (IAH) dividiendo el número total de apneas e hipopneas por el tiempo total de registro en horas. $\mathrm{La}$ ejecución e interpretación de los exámenes siguió las recomendaciones de la Academia Americana de Medicina del Sueño ${ }^{32,33}$. El diagnóstico y la gravedad del SAHOS se clasificó siguiendo las recomendaciones internacionales: registro normal (IAH $<5$ eventos/horas), SAHOS leve (IAH: 5-14,9), moderado (IAH: $15-29,9)$ y grave (IAH $\geq 30$ eventos/h).

\section{Análisis estadístico}

Los resultados fueron expresados como valores promedio \pm desviación estándar para las variables numéricas de distribución normal y en porcentaje para las medidas en escala nominal. Las variables de distribución desconocida fueron consignadas como mediana y rangos intercuartílicos. Las variables cualitativas fueron comparadas mediante la prueba de chi cuadrado y el test exacto de Fisher, y las variables continuas según su distribución con la prueba $t$ de Student o la prueba de Mann-Whitney. Para ello se utilizaron los programas estadísticos SPSS 25.0 y Stata/SE for large database. Las variables clínicas y las reglas predictivas asociadas al riesgo de SAHOS fueron sometidas a análisis univariado y multivariado en un modelo de regresión logística (modalidad stepwise) que permite el control simultáneo de múltiples factores. De este modo, los parámetros que no agregaron valor predictivo no fueron retenidos en el modelo. Se midió la sensibilidad, especificidad, valor predictivo positivo y negativo y las razones de probabilidades (odds ratio) de los diferentes predictores clínicos de SAHOS, con sus correspondientes intervalos de confianza (95\%). Las diferencias entre las variables fueron consideradas significativas con un valor de $\mathrm{p}<0,05$.

\section{Resultados}

En la Región Metropolitana, la ENS 2016/17 reclutó a 912 sujetos, edad media: $48 \pm 20$ años, $61,6 \%$ mujeres, un tercio fumadores activos, siendo las principales comorbilidades: hipertensión arterial (32,2\%), depresión (29,3\%), dislipidemia $(27,4 \%)$ y diabetes mellitus $(13,5 \%)$. En la historia de sueño, 57,5\% refieren ronquido habitual, $21,5 \%$ apneas presenciadas por testigos, $20,8 \%$ somnolencia diurna, $7,7 \%$ insomnio, $13,1 \%$ uso de hipnóticos y $40,5 \%$ sueño poco reparador. En la Tabla 1 se describe las características clínicas de la muestra de la Región Metropolitana y la submuestra de 205 sujetos a quienes se realizó 
Tabla 1. Características clínicas de la muestra de la Región Metropolitana de la Encuesta Nacional de Salud 2016/17 y de la submuestra de la Región Metropolitana a quienes se realizó poligrafía respiratoria en el hogar. Datos crudos, sin expansión muestral

\begin{tabular}{|c|c|c|c|c|}
\hline \multirow[b]{2}{*}{ Características } & \multicolumn{2}{|c|}{ Región Metropolitana } & \multicolumn{2}{|c|}{ Estudio del sueño en el hogar } \\
\hline & $\begin{array}{c}n \\
(X \pm D E)\end{array}$ & $\begin{array}{c}\text { Porcentaje } \\
\text { (Rango) }\end{array}$ & $\begin{array}{c}n \\
(X \pm D E)\end{array}$ & $\begin{array}{c}\text { Porcentaje } \\
\text { (Rango) }\end{array}$ \\
\hline N & 912 & & 205 & \\
\hline Edad (años) & $47,8 \pm 20,0$ & $15-94$ & $50,7 \pm 15,0$ & $18-84$ \\
\hline Género (masculino-femenino) & $350-562$ & $38,4-61,6$ & $95-110$ & $46,3-53,7$ \\
\hline Nivel educacional (A-B-C) & $172-458-280$ & $18,9-50,3-30,8$ & $28-108-69$ & $13,7-52,7-33,6$ \\
\hline $\begin{array}{l}\text { Consumo de tabaco } \\
\text { (fumador activo-exfumador-no fuma) }\end{array}$ & $296-282-334$ & $32,5-30,9-36,6$ & $67-82-56$ & $32,7-40,0-27,3$ \\
\hline Consumo de alcohol & $587 / 832$ & 70,6 & $154 / 205$ & 75,1 \\
\hline Menopausia & $202 / 431$ & 46,9 & $49 / 110$ & 44,5 \\
\hline Cardiopatía coronaria & $43 / 907$ & 4,7 & 15 & 7,3 \\
\hline Enfermedad cerebrovascular & $37 / 908$ & 4,1 & 12 & 5,8 \\
\hline Hipertensión arterial & $294 / 912$ & 32,2 & 77 & 37,6 \\
\hline Diabetes mellitus & $123 / 912$ & 13,5 & 35 & 17,1 \\
\hline Dislipidemia & $250 / 912$ & 27,4 & 88 & 42,9 \\
\hline Asma bronquial & $50 / 830$ & 6,0 & 12 & 5,9 \\
\hline Depresión & $266 / 909$ & 29,3 & 68 & 33,1 \\
\hline SAHOS & 19/832 & 2,3 & 10 & 4,8 \\
\hline Peso (kg) & $73,6 \pm 15,1$ & $30,6-146,9$ & $77,7 \pm 16,2$ & $48,1-146,9$ \\
\hline Talla (cm) & $160,9 \pm 9,6$ & $134,5-193$ & $162,3 \pm 9,7$ & $140-193$ \\
\hline IMC (kg/m²) & $28,4 \pm 5,2$ & $14,8-58,5$ & $29,4 \pm 5,3$ & $18,5-58,5$ \\
\hline Circunferencia cervical (cm) & & & $37,9 \pm 4,3$ & $29-55$ \\
\hline Circunferencia de cintura $(\mathrm{cm})$ & $93,3 \pm 13,8$ & $52,1-151,9$ & $96,2 \pm 13,4$ & $70,5-151,9$ \\
\hline Presión arterial sistólica (mmHg) & $124,3 \pm 21,8$ & $86-234$ & $124,9 \pm 18,3$ & $86-203$ \\
\hline Presión arterial diastólica (mmHg) & $73,6 \pm 10,3$ & $49-110$ & $75,2 \pm 9,6$ & $52-98$ \\
\hline Glicemia en ayunas (mg/dL) & $96,8 \pm 33,8$ & $64-498$ & $97,9 \pm 37,4$ & $72-442$ \\
\hline Colesterol sérico (mg/dL) & $177,7 \pm 40,6$ & $93-410$ & $184,9 \pm 46,1$ & $98-410$ \\
\hline Creatininemia (mg/dL) & $0,79 \pm 0,37$ & $0,39-7,37$ & $0,77 \pm 0,17$ & $0,45-1,29$ \\
\hline Duración del sueño (horas) & 6,9 & $3-12$ & 6,7 & $3-12$ \\
\hline Roncador habitual & $508 / 883$ & 57,5 & 157 & 76,6 \\
\hline Apneas presenciadas & $181 / 840$ & 21,5 & 103 & 50,2 \\
\hline Somnolencia diurna excesiva & $190 / 912$ & 20,8 & 56 & 27,3 \\
\hline Sueño poco reparador & $369 / 912$ & 40,5 & 96 & 46,8 \\
\hline Insomnio & $64 / 830$ & 7,7 & 21 & 10,2 \\
\hline Uso de hipnóticos & $119 / 911$ & 13,1 & 23 & 11,2 \\
\hline Escala de Epworth & $3,8 \pm 4,2$ & $0-21$ & $4,7 \pm 4,7$ & $0-21$ \\
\hline SAHOS leve-moderado-grave & & & $67-33-21$ & $32,7-16,1-10,2$ \\
\hline
\end{tabular}

Nota: $X \pm D E:$ Promedio \pm Desviación estándar; Nivel educacional: A: Menos de 8 años de estudio, B: 8-12 años, C: Más de 12 años de estudios; IMC: Índice de masa corporal; SAHOS: Síndrome de apneas-hipopneas obstructivas del sueño. 
poligrafía respiratoria en el hogar $(22,5 \%$ de la muestra de la Región Metropolitana).

En los 205 sujetos evaluados con cuestionario de sueño y poligrafía respiratoria en el hogar, 59\% tienen SAHOS (IA/H $>5$ eventos/h), siendo 26,3\% SAHOS moderado-grave (IA/H $\geq 15$ eventos/ hora). Las principales variables clínicas asociadas al riesgo de SAHOS fueron la edad, sexo masculino, hipertensión arterial, dislipidemia, IMC, circunferencia cervical y de cintura, presión arterial sistólica, historia de ronquido habitual y apneas presenciadas por testigos (Tablas 2 y 3 ). La som-

Tabla 2. Características clínicas y factores de riesgo de los sujetos con trastornos respiratorios del sueño (SAHOS) de la submuestra de la Región Metropolitana de la Encuesta Nacional de Salud 2016/17 a quienes se realizó poligrafía respiratoria en el hogar. Análisis univariado

\begin{tabular}{|c|c|c|c|c|c|}
\hline Características & SAHOS (-) & SAHOS (+) & $\mathbf{p}$ & $\begin{array}{c}\text { SAHOS } \\
\text { moderado-grave }\end{array}$ & $\mathbf{p}$ \\
\hline $\mathrm{N}$ & 84 & 121 & & 54 & \\
\hline Edad (años) & $44,4 \pm 15,4$ & $55,0 \pm 13,0$ & $<0,0001$ & $54,7 \pm 12,6$ & 0,0001 \\
\hline Género (masculino-femenino) & $33-51$ & $62-59$ & 0,0922 & $29-25$ & 0,0978 \\
\hline Nivel educacional (A-B-C) & $6-46-32$ & $22-62-37$ & 0,0681 & $10-29-15$ & 0,0952 \\
\hline Actividad física & 14 & 14 & 0,2972 & 6 & 0,3673 \\
\hline Consumo de tabaco & 34 & 33 & 0,0480 & 16 & 0,1974 \\
\hline Consumo de alcohol & 67 & 87 & 0,2015 & 41 & 0,5952 \\
\hline Cardiopatía coronaria & 5 & 10 & 0,5329 & 7 & 0,1552 \\
\hline Enfermedad cerebrovascular & 4 & 8 & 0,5800 & 3 & 0,8363 \\
\hline Hipertensión arterial & 23 & 54 & 0,0124 & 26 & 0,0132 \\
\hline Diabetes mellitus & 15 & 20 & 0,8042 & 13 & 0,3772 \\
\hline Dislipidemia & 25 & 63 & 0,0015 & 26 & 0,0296 \\
\hline Asma bronquial & 8 & 4 & 0,0628 & 3 & 0,4026 \\
\hline EPOC & 3 & 3 & 0,6490 & 2 & 0,9677 \\
\hline Depresión & 24 & 44 & 0,2450 & 20 & 0,2994 \\
\hline $\operatorname{IMC}\left(\mathrm{kg} / \mathrm{m}^{2}\right)$ & $27,8 \pm 6,1$ & $30,6 \pm 4,3$ & 0,0002 & $31,3 \pm 4,7$ & 0,0005 \\
\hline Circunferencia cervical (cm) & $36,4 \pm 4,6$ & $38,9 \pm 3,7$ & $<0,0001$ & $39,1 \pm 3,7$ & 0,0004 \\
\hline Circunferencia de cintura $(\mathrm{cm})$ & $90,9 \pm 14,6$ & $99,9 \pm 11,1$ & $<0,0001$ & $102,3 \pm 12,2$ & $<0,0001$ \\
\hline Presión arterial sistólica (mmHg) & $119,4 \pm 16,0$ & $128,8 \pm 18,8$ & 0,0002 & $127,9 \pm 16,7$ & 0,0033 \\
\hline Presión arterial diastólica $(\mathrm{mmHg})$ & $74,2 \pm 10,1$ & $76,0 \pm 9,2$ & 0,1872 & $76,0 \pm 9,9$ & 0,3050 \\
\hline Glicemia (mg/dL) & $95,2 \pm 38,3$ & $99,8 \pm 36,8$ & 0,3877 & $106,2 \pm 51,7$ & 0,1542 \\
\hline Colesterol total (mg/dL) & $172,8 \pm 37,3$ & $193,1 \pm 49,9$ & 0,0018 & $187,3 \pm 59,1$ & 0,0793 \\
\hline Duración del sueño (horas) & $6,7 \pm 1,6$ & $6,8 \pm 1,8$ & 0,6829 & $6,9 \pm 1,7$ & 0,4856 \\
\hline Roncador habitual & 56 & 101 & 0,0053 & 46 & 0,0160 \\
\hline Apneas presenciadas & 32 & 71 & 0,0038 & 32 & 0,0153 \\
\hline Somnolencia diurna excesiva & 21 & 35 & 0,5360 & 17 & 0,4072 \\
\hline Sueño poco reparador & 43 & 53 & 0,2983 & 23 & 0,3255 \\
\hline Insomnio & 12 & 9 & 0,1127 & 5 & 0,3823 \\
\hline Uso de hipnóticos & 11 & 12 & 0,4794 & 7 & 0,9821 \\
\hline Cuestionario STOP & $1,6 \pm 1,2$ & $2,2 \pm 1,0$ & 0,0001 & $2,2 \pm 1,0$ & 0,0027 \\
\hline Cuestionario STOP-Bang & $2,6 \pm 1,5$ & $3,9 \pm 1,4$ & $<0,0001$ & $4,0 \pm 1,3$ & $<0,0001$ \\
\hline Escala de Epworth & $4,0 \pm 4,8$ & $5,4 \pm 4,6$ & 0,0365 & $5,7 \pm 4,8$ & 0,0443 \\
\hline Índice de apneas-hipopneas & $2,4 \pm 1,3$ & $18,6 \pm 14,5$ & $<0,0001$ & $30,1 \pm 15,0$ & $<0,0001$ \\
\hline
\end{tabular}

Nota: Nivel educacional: A: Menos de 8 años de estudio, B: 8-12 años, C: Más de 12 años de estudios; EPOC: Enfermedad pulmonar obstructiva crónica; IMC: Índice de masa corporal; SAHOS: Síndrome de apneas-hipopneas obstructivas del sueño. 


\begin{abstract}
Tabla 3. Factores de riesgo, cuestionarios de sueño y variables poligráficas de la muestra de la Región Metropolitana de la Encuesta Nacional de Salud 2016/17 a quienes se realizó poligrafía respiratoria en el hogar
\end{abstract}

\begin{tabular}{|c|c|c|c|}
\hline Características & SAHOS (-) & SAHOS moderado-grave & $\mathbf{p}$ \\
\hline $\mathrm{N}$ & 84 & 54 & \\
\hline Roncador-apneas & $30 / 84(35,7 \%)$ & $32 / 54(59,3 \%)$ & 0,0069 \\
\hline Roncador-apneas-SDE & $12 / 84(14,3 \%)$ & $11 / 54(20,4 \%)$ & 0,3510 \\
\hline $\mathrm{IMC}>30 \mathrm{~kg} / \mathrm{m}^{2}$ & $17 / 82(20,7 \%)$ & $33 / 52(63,5 \%)$ & $<0,0001$ \\
\hline Circunferencia cervical $>36 \mathrm{~cm}$ & $36 / 84(42,8 \%)$ & $38 / 50(76 \%)$ & 0,0002 \\
\hline Circunferencia de cintura $>94 \mathrm{~cm}$ & $25 / 82(30,5 \%)$ & $41 / 52(78,8 \%)$ & $<0,0001$ \\
\hline STOP: Alto riesgo de SAHOS & $39 / 84(46,4 \%)$ & $41 / 54(75,9 \%)$ & 0,0011 \\
\hline STOP-Bang: Alto riesgo de SAHOS & $46 / 84(54,8 \%)$ & $48 / 54(88,9 \%)$ & $<0,0001$ \\
\hline Escala de Epworth: Somnolencia diurna excesiva & $7 / 84(8,3 \%)$ & $10 / 54(18,5 \%)$ & 0,0767 \\
\hline Accidentes de tránsito por SDE & $0 / 51(0 \%)$ & $1 / 42(2,4 \%)$ & 0,2705 \\
\hline Duración registro de PR (horas) & $6,0 \pm 1,5$ & $6,2 \pm 1,3$ & 0,4226 \\
\hline Duración del ronquido (\% tiempo de registro) & $10,3 \pm 15,7$ & $20,4 \pm 17,5$ & 0,0006 \\
\hline $\mathrm{SaO}_{2}$ basal $(\%)$ & $96,8 \pm 1,6$ & $96,1 \pm 1,7$ & 0,0157 \\
\hline $\mathrm{SaO}_{2}$ media (\%) & $94,1 \pm 1,7$ & $91,5 \pm 2,2$ & $<0,0001$ \\
\hline $\mathrm{SaO}_{2}$ mínima (\%) & $87,1 \pm 4,4$ & $77,0 \pm 6,1$ & $<0,0001$ \\
\hline CT-90\% (\%) & $1,7 \pm 5,4$ & $17,4 \pm 19,7$ & $<0,0001$ \\
\hline ID4\% & $2,2 \pm 1,6$ & $23,5 \pm 13,9$ & $<0,0001$ \\
\hline Frecuencia cardiaca media (lat/min) & $66,7 \pm 10,4$ & $67,2 \pm 10,5$ & 0,7840 \\
\hline Duración media apneas (seg) & $15,5 \pm 7,8$ & $19,2 \pm 4,8$ & 0,0022 \\
\hline Duración media hipopneas (seg) & $21,3 \pm 5,4$ & $24,7 \pm 5,8$ & 0,0006 \\
\hline Índice de apneas-hipopneas & $2,4 \pm 1,3$ & $30,1 \pm 15,0$ & $<0,0001$ \\
\hline Índice de apneas-hipopneas DS & $3,2 \pm 2,7$ & $34,0 \pm 19,3$ & $<0,0001$ \\
\hline Índice de apneas-hipopneas DL & $2,2 \pm 4,1$ & $17,4 \pm 19,1$ & $<0,0001$ \\
\hline
\end{tabular}

Nota: $\mathrm{X} \pm \mathrm{DE}$ : Promedio \pm Desviación estándar; SDE: Somnolencia diurna excesiva; IMC: Índice de masa corporal; SAHOS: Síndrome de apneas-hipopneas obstructivas del sueño; PR: Poligrafía respiratoria; $\mathrm{SaO}_{2}$ : Saturación arterial de oxígeno; CT90\%: Porcentaje del tiempo con $\mathrm{SaO}_{2}$ bajo 90\%; ID 4\%: Índice de desaturación de 4\%; DS: Decúbito supino; DL: Decúbito lateral.

nolencia diurna, insomnio y sueño poco reparador no se asociaron al riesgo de SAHOS. En el análisis multivariado, las principales variables clínicas asociadas al riesgo de SAHOS fueron la edad, sexo masculino, reporte de apneas, insomnio, diabetes mellitus, dislipidemia y el IMC (Tabla 4).

Los principales predictores clínicos de SAHOS fueron la edad, historia de ronquido y pausas respiratorias durante el sueño, IMC, circunferencia cervical y de cintura elevados (Tabla 5). Los cues- tionarios de sueño STOP y STOP-Bang se asociaron al riesgo de SAHOS en la muestra poblacional examinada en la Región Metropolitana (AUC: 0,65 y $0,72, p<0,001$, respectivamente). La escala de somnolencia de Epworth se asoció discretamente al riesgo de SAHOS (AUC: 0,59, IC 95\% 0,52-0,66, p: 0,026$)$. Los cuestionarios STOP y STOP-Bang fueron más sensibles y la escala de Epworth fue más específica en la pesquisa de pacientes con SAHOS (Tabla 6). 
Cuestionarios en apneas obstructivas del sueño - F. Saldías Peñafiel et al

Tabla 4. Variables clínicas asociadas al riesgo de síndrome de apneas obstructivas del sueño. Análisis multivariado

\begin{tabular}{|lccccc|}
\hline & Coeficiente & Error estándar & Odds ratio & IC 95\% & p \\
Riesgo de SAHOS & & & & & \\
$\quad$ Edad & 0,0719 & 0,0149 & 1,08 & $1,04-1,11$ & $<0,0001$ \\
Sexo masculino & 1,2778 & 0,4196 & 3,59 & $1,58-8,17$ & 0,0023 \\
Apneas presenciadas & 1,2527 & 0,3937 & 3,50 & $1,62-7,57$ & 0,0015 \\
Insomnio & $-1,2644$ & 0,6401 & 0,28 & $0,08-0,99$ & 0,0482 \\
Diabetes mellitus & $-1,5281$ & 0,5291 & 0,22 & $0,08-0,61$ & 0,0039 \\
Dislipidemia & 0,9314 & 0,4032 & 2,54 & $1,15-5,59$ & 0,0209 \\
Depresión & 0,8718 & 0,4694 & 2,39 & $0,95-6,00$ & 0,0633 \\
IMC & 0,1516 & 0,0397 & 1,16 & $1,08-1,26$ & 0,0001 \\
Riesgo de SAHOS moderado-grave & & & & & \\
Edad & 0,0299 & 0,0123 & 1,03 & $1,01-1,06$ & 0,0155 \\
Sexo masculino & 0,6427 & 0,3544 & 1,90 & $0,95-3,81$ & 0,0698 \\
IMC & 0,1002 & 0,0332 & 1,11 & $1,04-1,18$ & 0,0026 \\
\hline
\end{tabular}

Tabla 5. Evaluación del área bajo la curva receptor operador (AUC) de los predictores clínicos y cuestionarios de sueño en el diagnóstico de trastornos respiratorios del sueño (SAHOS)

\begin{tabular}{|llc|}
\hline Predictores clínicos & SAHOS AUC (IC 95\%, p) & SAHOS moderado-grave AUC (IC 95\%, p) \\
\hline Edad & $0,69(0,63-0,76, p<0,0001)$ & $0,59(0,52-0,66, p: 0,0371)$ \\
\hline Roncador-apneas & $0,61(0,54-0,68, p: 0,0014)$ & $0,57(0,50-0,64, p: 0,0709)$ \\
\hline Roncador-apneas-SDE & $0,52(0,45-0,59, p: 0,3685)$ & $0,52(0,45-0,59, p: 0,4764)$ \\
Roncador-apnea-IMC $>30$ & $0,61(0,54-0,68, p<0,0001)$ & $0,60(0,53-0,67, p: 0,0067)$ \\
Índice de masa corporal & $0,73(0,66-0,78, p<0,0001)$ & $0,68(0,61-0,74, p<0,0001)$ \\
Circunferencia cervical & $0,67(0,60-0,74, p<0,0001)$ & $0,62(0,54-0,68, p: 0,0079)$ \\
Circunferencia de cintura & $0,74(0,67-0,79, p<0,0001)$ & $0,70(0,63-0,76, p<0,0001)$ \\
Cuestionario STOP & $0,65(0,58-0,71, p: 0,0002)$ & $0,60(0,53-0,67, p: 0,0141)$ \\
\hline Cuestionario STOP-Bang & $0,72(0,65-0,78, p<0,0001)$ & $0,67(0,60-0,73, p<0,0001)$ \\
Escala de Epworth & $0,59(0,52-0,66, p: 0,0263)$ & $0,58(0,51-0,65 p: 0,0628)$ \\
\hline
\end{tabular}

Nota: SAHOS: Síndrome de apneas hipopneas obstructivas del sueño, AUC: Área bajo la curva receptor operador, IC 95\%: Intervalo de confianza de 95\%; SDE: Somnolencia diurna excesiva; IMC: Índice de masa corporal.

Tabla 6. Sensibilidad, especificidad, valores predictivos y odds ratio de las categorías de alto riesgo de los cuestionarios de sueño en el diagnóstico de trastornos respiratorios del sueño (SAHOS moderado-grave)

\begin{tabular}{|lccccccc|}
\hline Cuestionarios & $\begin{array}{c}\text { Sensi- } \\
\text { bilidad }\end{array}$ & $\begin{array}{c}\text { Especi- } \\
\text { ficidad }\end{array}$ & VPP & VPN & $\begin{array}{c}\text { Odds } \\
\text { ratio }\end{array}$ & IC 95\% & p \\
\hline Roncador-apneas & 0,59 & 0,55 & 0,32 & 0,79 & 1,78 & $0,95-3,34$ & 0,0743 \\
\hline Roncador-apneas-IMC $>30 \mathrm{~kg} / \mathrm{m}^{2}$ & 0,37 & 0,83 & 0,44 & 0,79 & 2,83 & $1,41-5,66$ & 0,0034 \\
\hline Circunferencia cervical $>35 \mathrm{~cm}$ & 0,84 & 0,37 & 0,31 & 0,87 & 3,14 & $1,37-7,17$ & 0,0067 \\
\hline Circunferencia de cintura $>94 \mathrm{~cm}$ & 0,79 & 0,52 & 0,37 & 0,87 & 3,99 & $1,90-8,36$ & 0,0002 \\
\hline STOP & 0,76 & 0,40 & 0,31 & 0,82 & 2,08 & $1,03-4,20$ & 0,0415 \\
\hline STOP-Bang & 0,89 & 0,36 & 0,33 & 0,90 & 4,45 & $1,79-11,1$ & 0,0013 \\
\hline Epworth & 0,19 & 0,91 & 0,42 & 0,76 & 2,22 & $0,92-5,36$ & 0,0749 \\
\hline
\end{tabular}

Nota: VPP: Valor predictivo positivo, VPN: Valor predictivo negativo, IC 95\%: Intervalo de confianza de $95 \%$. 


\section{Discusión}

En este estudio examinamos las principales variables clínicas asociadas al riesgo de SAHOS y el rendimiento de los cuestionarios de sueño en una muestra poblacional seleccionada de la Región Metropolitana de la Encuesta Nacional de Salud 2016/17 a quienes se realizó cuestionario de sueño, mediciones antropométricas y poligrafía respiratoria en el hogar.

En nuestro estudio, las principales variables clínicas asociadas al riesgo de SAHOS fueron la edad, sexo masculino, hipertensión arterial, dislipidemia, obesidad de predominio abdominal o visceral objetivada mediante el IMC, circunferencia cervical y de cintura, historia de ronquido habitual y reporte de pausas respiratorias durante el sueño. Estas variables clínicas son incluidas en los principales cuestionarios de sueño empleados en la pesquisa de pacientes con trastornos respiratorios del sueño, tales como el índice de Flemons, cuestionario de Berlín, STOP, STOP-Bang, cuestionario de sueño de Wisconsin e índice de calidad de sueño de Pittsburgh, entre otros ${ }^{17,18,20,21-23}$. La historia de ronquido intenso y de larga data tiene elevada sensibilidad pero baja especificidad para la pesquisa de pacientes con trastornos respiratorios del sueño; destacando que alrededor de la mitad de los hombres $(59,9 \%)$ y mujeres $(51,1 \%)$ refirieron ser roncadores habituales en la encuesta nacional de salud ${ }^{34}$. Similar a lo descrito en otros estudios, las apneas o pausas respiratorias presenciadas por testigos constituyen el hallazgo más específico en la historia clínica ${ }^{13-16,21-23}$. La historia de somnolencia diurna excesiva, insomnio, uso de hipnóticos y sueño poco reparador no se asociaron al riesgo de SAHOS en la muestra poblacional. La somnolencia diurna excesiva objetivada mediante la escala de somnolencia de Epworth no nos permitió identificar a los pacientes con SAHOS moderado-grave, lo cual es explicable por la elevada prevalencia de este síntoma en la población general, la variable percepción del síntoma según la edad y sexo de la población, y su amplio diagnóstico diferencial ${ }^{35-37}$. En la encuesta nacional de salud 2016/17, el 18,4\% de los sujetos encuestados reportaron somnolencia diurna excesiva al menos tres días a la semana, síntoma reportado por el $16,4 \%$ de los varones y $19,6 \%$ de las mujeres. Por este motivo, las escalas de somnolencia empleadas en los estudios poblacionales y clínicas de sueño no han permitido identificar con precisión a los pacientes portadores de trastornos respiratorios del sueño $0^{19,38-40}$.

En el examen físico, los principales hallazgos clínicos asociados al riesgo de SAHOS fueron la pesquisa de cifras tensionales elevadas y sobrepeso u obesidad de predominio visceral objetivados mediante el IMC, circunferencia cervical y de cintura (Tablas 2-4). A diferencia de otros estudios ${ }^{17,21,41-47}$, el índice de masa corporal y la circunferencia de cintura fueron mejores predictores de SAHOS comparado con la circunferencia cervical. En esta misma línea, Deegan y cols., encontraron que la circunferencia de cintura era mejor predictor del índice de apneas e hipopneas comparado con el perímetro cervical en varones y lo opuesto aconteció en las mujeres ${ }^{16}$. En el análisis multivariado, los principales predictores clínicos de SAHOS fueron la edad, sexo masculino, apneas presenciadas e índice de masa corporal; variables clínicas incluidas en el índice de Flemons ${ }^{17}$ y cuestionario STOP-Bang ${ }^{21}$. La historia de ronquido regular y apneas presenciadas asociado a obesidad es bastante específico y orientador en el diagnóstico de SAHOS, sin embargo, es poco sensible. En cambio, el perímetro cervical y de cintura elevados son bastante sensibles, pero inespecíficos en la pesquisa de pacientes con SAHOS moderado-grave, quienes más se beneficiarán del diagnóstico y tratamiento oportuno.

En nuestro estudio, los cuestionarios de sueño STOP y STOP-Bang permitieron predecir con bastante precisión el riesgo de SAHOS en la muestra poblacional de la Región Metropolitana (Tabla 5). El desempeño de los cuestionarios de sueño fue variable, con áreas bajo la curva receptor operador que fluctuaron entre 0,59 y 0,72 ; los cuestionarios STOP y STOP-Bang fueron más sensibles y la escala de somnolencia de Epworth fue más específica en la pesquisa de pacientes con SAHOS. Los cuestionarios de sueño STOP y STOP-Bang tuvieron elevado valor predictivo negativo pero bajo valor predictivo positivo, similar a lo descrito en otros estudios $^{28}$. Por este motivo, los cuestionarios STOP y STOP-Bang han sido ampliamente empleados en la pesquisa de pacientes con SAHOS en diferentes contextos clínicos: servicios de atención primaria, evaluación preoperatoria, estudios poblacionales y clínicas de sueño ${ }^{19,20,24}$.

En este estudio poblacional realizado en una submuestra de la Región Metropolitana destaca la elevada prevalencia de trastornos respiratorios 
del sueño en población adulta, siendo $26,3 \%$ de magnitud moderada a severa; es decir, el grupo de mayor riesgo que se beneficiaría de la pesquisa y tratamiento oportuno, mejorando su calidad de vida, riesgo cardiovascular y sobrevida a mediano y largo plazo. Las principales variables clínicas asociadas al riesgo de SAHOS fueron la edad, sexo masculino, hipertensión arterial, dislipidemia y obesidad de distribución central $\mathrm{u}$ abdominal. La historia de ronquido regular, apneas presenciadas por testigos y obesidad es bastante específico para el diagnóstico de esta condición. Los cuestionarios de sueño fueron bastante sensibles para la pesquisa de pacientes con trastornos respiratorios del sueño moderados y graves en esta muestra poblacional, lo cual valida su empleo en los estudios de prevalencia de SAHOS realizados en la población adulta.

\section{Referencias}

1. Usmani ZA, Chai-Coetzer CL, Antic NA, McEvoy RD. Obstructive sleep apnoea in adults. Postgrad Med J 2013; 89: 148-56.

2. Farrell PC, Richards G. Recognition and treatment of sleep-disordered breathing: an important component of chronic disease management. J Transl Med 2017; 15: 114.

3. Laratta CR, Ayas NT, Povitz M, Pendharkar SR. Diagnosis and treatment of obstructive sleep apnea in adults. CMAJ 2017; 189: E1481-8.

4. Lin J, Suurna M. Sleep Apnea and Sleep-Disordered Breathing. Otolaryngol Clin North Am 2018; 51: 827-33.

5. Franklin KA, Lindberg E. Obstructive sleep apnea is a common disorder in the population-a review on the epidemiology of sleep apnea. J Thoracic Dis 2015; 7: 1311-22.

6. Senaratna CV, Perret JL, Lodge CJ, Lowe AJ, Campbell $\mathrm{BE}$, Matheson MC, et al. Prevalence of obstructive sleep apnea in the general population: A systematic review. Sleep Med Rev 2017; 34: 70-81.

7. Wang AY. Sleep-disordered breathing and resistant hypertension. Semin Nephrol 2014; 34: 520-31.

8. Wolf J, Narkiewicz K. Optimizing the management of uncontrolled/resistant hypertension. The Importance of sleep apnoea syndrome. Curr Vasc Pharmacol 2017; 16: 44-53.

9. Hla KM, Young T, Hagen EW, Stein JH, Finn LA, Nieto FJ, et al. Coronary heart disease incidence in sleep disordered breathing: the Wisconsin Sleep Cohort Study. Sleep 2015; 38: 677-84.
10. Goudis CA, Ketikoglou DG. Obstructive sleep and atrial fibrillation: Pathophysiological mechanisms and therapeutic implications. Int J Cardiol 2017; 230: 293-300.

11. Redline S, Yenokyan G, Gottlieb DJ, Shahar E, O'Connor GT, Resnick HE, et al. Obstructive sleep apnea-hypopnea and incident stroke: the sleep heart health study. Am J Respir Crit Care Med 2010; 182: 269-77.

12. Drager LF, Togeiro SM, Polotsky VY, Lorenzi-Filho G. Obstructive sleep apnea: a cardiometabolic risk in obesity and the metabolic syndrome. J Am Coll Cardiol 2013; 62: 569-76.

13. Viner S, Szalai JP, Hoffstein V. Are history and physical examination a good screening test for sleep apnea? Ann Intern Med 1991; 115: 356-9.

14. Hoffstein V, Szalai JP. Predictive value of clinical features in diagnosing obstructive sleep apnea. Sleep 1993; 16: 118-22.

15. Kump K, Whalen C, Tishler PV, Browner I, Ferrette V, Strohl KP, et al. Assessment of the validity and utility of a sleep-symptom questionnaire. Am J Respir Crit Care Med 1994; 150: 735-41.

16. Deegan PC, McNicholas WT. Predictive value of clinical features for the obstructive sleep apnoea syndrome. Eur Respir J 1996; 9: 117-24.

17. Flemons WW, McNicholas WT. Clinical prediction of the sleep apnea syndrome. Sleep Med Rev 1997; 1: 1932.

18. Netzer NC, Stoohs RA, Netzer CM, Clark K, Strohl KP. Using the Berlin Questionnaire to identify patients at risk for the sleep apnea syndrome. Ann Intern Med 1999; 131: 485-91.

19. Sil A, Barr G. Assessment of predictive ability of Epworth scoring in screening of patients with sleep apnoea. J Laryngol Otol 2012; 126: 372-9.

20. Chung F, Yegneswaran B, Liao P, Chung SA, Vairavanathan S, Islam S. STOP questionnaire: a tool to screen patients for obstructive sleep apnea. Anesthesiology 2008; 108: 812-21.

21. Chung F, Abdullah HR, Liao P. STOP-Bang Questionnaire: A practical approach to screen for obstructive sleep apnea. Chest 2016; 149: 631-8.

22. Abrishami A, Khajehdehi A, Chung F. A systematic review of screening questionnaires for obstructive sleep apnea. Can J Anaesth 2010; 57: 423-38.

23. Fedson AC, Pack AI, Gislason T. Frequently used sleep questionnaires in epidemiological and genetic research for obstructive sleep apnea: a review. Sleep Med Rev 2012; 16: 529-37.

24. Kapur VK, Auckley DH, Chowdhuri S, Kuhlmann DC, Mehra R, Ramar K, et al. Clinical Practice Guideline for Diagnostic Testing for Adult Obstructive Sleep Apnea: 
An American Academy of Sleep Medicine Clinical Practice Guideline. J Clin Sleep Med 2017; 13: 479-504.

25. Bouscoulet LT, Vázquez-García JC, Muiño A, Márquez M, López MV, de Oca MM, et al; PLATINO Group. Prevalence of sleep related symptoms in four Latin American cities. J Clin Sleep Med 2008; 4: 579-85.

26. Encuesta Nacional de Salud ENS Chile 2009-2010. Ministerio de Salud, Chile. Páginas 496-500.

27. Johns MW. A new method for measuring daytime sleepiness: the Epworth sleepiness scale. Sleep 1991; 14: 540-5.

28. Nagappa M, Liao P, Wong J, Auckley D, Ramachandran SK, Memtsoudis S, et al. Validation of the STOP-Bang questionnaire as a screening tool for obstructive sleep apnea among different populations: A systematic review and meta-analysis. PLoS One 2015; 10: e0143697.

29. Collop NA, Anderson WM, Boehlecke B, Claman D, Goldberg R, Gottlieb DJ, et al. Clinical guidelines for the use of unattended portable monitors in the diagnosis of obstructive sleep apnea in adult patients. Portable Monitoring Task Force of the American Academy of Sleep Medicine. J Clin Sleep Med 2007; 3: 737-47.

30. El Shayeb M, Topfer LA, Stafinski T, Pawluk L, Menon D. Diagnostic accuracy of level 3 portable sleep tests versus level 1 polysomnography for sleep-disordered breathing: a systematic review and meta-analysis. CMAJ 2014; 186: E25-51.

31. Lux L, Boehlecke B, Lohr KN. Effectiveness of Portable Monitoring Devices for Diagnosing Obstructive Sleep Apnea: Update of a Systematic Review. Rockville (MD): Agency for Healthcare Research and Quality (US); 2004. AHRQ Technology Assessments.

32. Berry RB, Budhiraja R, Gottlieb DJ, Gozal D, Iber C, Kapur VK, et al. Rules for scoring respiratory events in sleep: update of the 2007 AASM Manual for the Scoring of Sleep and Associated Events. Deliberations of the Sleep Apnea Definitions Task Force of the American Academy of Sleep Medicine. J Clin Sleep Med 2012; 8: 597-619.

33. Berry RB, Albertario CL, Harding SM, Lloyd RM, Quan SF, Vaughn BV, et al; for the American Academy of Sleep Medicine. The AASM Manual for the Scoring of Sleep and Associated Events: Rules, Terminology and Technical Specifications. Version 2.5. Darien, IL: American Academy of Sleep Medicine; 2018.

34. Encuesta Nacional de Salud 2016-2017. Departamento de Epidemiología. Ministerio de Salud de Chile. http:// epi.minsal.cl/encuesta-nacional-de-salud-2015-2016/

35. Kapur VK, Baldwin CM, Resnick HE, Gottlieb DJ, Nieto FJ. Sleepiness in patients with moderate to severe sleep-disordered breathing. Sleep 2005; 28: 472-7.

36. Roure N, Gómez S, Mediano O, Durán J, Peña M de L, Capote F, et al. Daytime sleepiness and polysomnography in obstructive sleep apnea patients. Sleep Med 2008; 9: 727-31.

37. Parkes JD. ABC of sleep disorders. Daytime sleepiness. BMJ 1993; 306: 772-5.

38. Kingshott RN, Sime PJ, Engleman HM, Douglas NJ. Self assessment of daytime sleepiness: patient versus partner. Thorax 1995; 50: 994-5.

39. Osman EZ, Osborne J, Hill PD, Lee BW. The Epworth Sleepiness Scale: can it be used for sleep apnoea screening among snorers? Clin Otolaryngol Allied Sci 1999; 24: 239-41.

40. Rosenthal LD, Dolan DC. The Epworth sleepiness scale in the identification of obstructive sleep apnea. J Nerv Ment Dis 2008; 196: 429-31.

41. Katz I, Stradling J, Slutsky AS, Zamel N, Hoffstein V. Do patients with obstructive sleep apnea have thick necks? Am Rev Respir Dis 1990; 141 (5 Pt 1): 1228-31.

42. Davies RJ, Stradling JR. The relationship between neck circumference, radiographic pharyngeal anatomy, and the obstructive sleep apnoea syndrome. Eur Respir J 1990; 3: 509-14.

43. Stradling JR, Crosby JH. Predictors and prevalence of obstructive sleep apnoea and snoring in 1001 middle aged men. Thorax 1991; 46: 85-90.

44. Hoffstein V, Mateika S. Differences in abdominal and neck circumferences in patients with and without obstructive sleep apnoea. Eur Respir J 1992; 5: 377-81.

45. Davies RJ, Ali NJ, Stradling JR. Neck circumference and other clinical features in the diagnosis of the obstructive sleep apnoea syndrome. Thorax 1992; 47: 101-5.

46. Dixon JB, Schachter LM, O'Brien PE. Predicting sleep apnea and excessive day sleepiness in the severely obese: indicators for polysomnography. Chest 2003; 123: 113441.

47. Martinho FL, Tangerina RP, Moura SM, Gregório LC, Tufik S, Bittencourt LR. Systematic head and neck physical examination as a predictor of obstructive sleep apnea in class III obese patients. Braz J Med Biol Res 2008; 41: 1093-7. 